\title{
ON SOLVABILITY OF THE BVP FORMULATED IN TERMS OF DISPLACEMENT ORIENTATIONS ON THE INTERFACE BETWEEN DISSIMILAR ELASTIC MATERIALS
}

\author{
A.N. GALYBIN \\ The Schmidt Institute of Physics of the Earth (IPE RAS), Moscow, Russia.
}

\begin{abstract}
This article addresses a specific type of boundary conditions in plane elastic boundary value problems, BVP. An elastic plane composed of two dissimilar isotropic materials is considered. It is assumed that the displacement vector orientations are known on both sides of the contour that separates the entire plane into interior and exterior domains. The stress vector is assumed to be continuous across the contour.

The aim of this study is the investigation of solvability of this BVP and the development of appropriate numerical methods for solving the corresponding singular integral equation. The latter is necessary as the integral equation is homogeneous.

It is shown that depending on the behaviour of the displacement vector orientations the solution of the problem may include a certain number of arbitrary linear parameters. A numerical approach is proposed based on the solution of the homogeneous Riemann BVP to form a non-homogeneous right hand side of the integral equation.

Keywords: boundary value problems, complex potentials, plane elasticity, singular integral equations.
\end{abstract}

\section{INTRODUCTION}

Gakhov [1] investigated solvability of a special singular integral equation, SIE, which dominant part includes an unknown function $g$ and its conjugate $\bar{g}$ and the singular integral and its conjugate. In general this SIE can be presented in the following form

$$
a g+b \bar{g}+c \mathbf{S} g+d \overline{\mathbf{S} g}+\mathbf{K} g+\overline{\mathbf{L} g}=f
$$

Here $\mathbf{S g}$ is the singular operator

$$
\mathbf{S} g=\frac{1}{\pi i} \int_{\Gamma} \frac{g(\eta) d \eta}{\eta-\zeta}
$$

$\mathbf{K g}$ and $\mathbf{L g}$ are any regular operators and $a, b, c, d, f$ are known continuous functions of the complex contour variable. It has been shown that the solvability of SIE (1) is determined by the index calculated through the increment of the argument of the complex valued function $G$ after the complete traverse of $\Gamma$ in the counter-clockwise direction

$$
\begin{aligned}
& 2 \mathrm{~K} \equiv \operatorname{Index}(G)=\left.\frac{1}{2 \pi i}[\ln (G)]\right|_{\Gamma}=\left.\frac{1}{2 \pi}[\arg (G)]\right|_{\Gamma} \\
& G=\frac{A-i B}{A+i B}, \quad A-i B=(\bar{a}+\bar{c})(a-c)+(b+d)(\bar{d}-\bar{b})
\end{aligned}
$$

If $2 \mathrm{~K}>0$ (the coefficient 2 is introduced for convenience), then the solution of the homogeneous equation SIE $(f=0)$ depends on $2 \mathrm{~K}$ complex constants or $4 \mathrm{~K}$ real constants, otherwise the SIE does not have solutions, see Gakhov [1].

Galybin [2] has investigated solvability of four boundary value problems, BVPs, formulated in terms of the displacement and traction orientations on the contour that separates homogeneous elastic plane into interior and exterior domains. Such BVPs have been reduced 
to the form of SIE in (1), which allowed for the application of the Gakhov's approach. Investigation of the solvability of such SIEs, follows the idea proposed by Gakhov in the third Russian edition of his book (Boundary Value Problems, 1977), but not included in earlier editions translated into English (see [3] for details).

It should be mentioned that such types of BVPs are not only of academic interest. They find applications in geodynamics where the data on displacements are known only for the period of observations (e.g. from GPS observations or geological studies) and therefore their full magnitudes remain unknown, while the data on directions are more reliable. Therefore the motivation of the present study is to accommodate this specific information for the sake of stress analysis in tectonic regions. It should be noted that the case considered further is not covered in [3] as we consider dissimilar materials that form the entire plane. This is also important for geodynamics as the adjacent tectonic plates can have different elastic properties.

\section{SINGULAR INTEGRAL EQUATION OF THE PROBLEM}

\subsection{Problem formulation in terms of complex potentials}

The general solution of plane elastic boundary value problems is given by the Kolosov-Muskhelishvili formulas [3] in terms of two holomorphic functions (complex potentials) $\varphi(z)$ and $\psi(z)$ of complex variable $z=x_{1}+i x_{2}$. Let $\Gamma$ be a closed contour separating the entire complex plane into interior $\boldsymbol{\Omega}^{+}$and exterior $\boldsymbol{\Omega}^{-}$domains composed of dissimilar materials that, in general, have different elastic properties, $G^{ \pm}$(shear moduli) and $\boldsymbol{v}^{ \pm}$(Poisson's ratios).

The boundary values of displacements, $\mathbf{w}=\left(w_{1}, w_{2}\right)$, and tractions, $\mathbf{t}=\left(t_{1}, t_{2}\right)$, can be presented as complex valued functions (of complex variable $\zeta \in \Gamma$ ) $W$ and $T$ respectively via the boundary values of complex potentials

$$
\begin{aligned}
& 2 G^{ \pm} W^{ \pm}=2 G^{ \pm}\left(w_{1}^{ \pm}+i w_{2}^{ \pm}\right)=\kappa^{ \pm} \varphi^{ \pm}-\overline{\mathrm{H}}^{ \pm} \\
& T^{ \pm}=t_{1}^{ \pm}+i t_{2}^{ \pm}=\varphi^{ \pm}+\overline{\mathrm{H}}^{ \pm}
\end{aligned}
$$

Hereafter the symbols " \pm " denote the boundary values obtained by approaching $\Gamma$ from domains $\boldsymbol{\Omega}^{ \pm}$respectively; piecewise elastic constants $\boldsymbol{\kappa}^{ \pm}$are defined as $\boldsymbol{\kappa}^{ \pm}=3-4 \boldsymbol{v}^{ \pm}$to for plane strain and $\boldsymbol{\kappa}^{ \pm}=\left(3-\boldsymbol{v}^{ \pm}\right)\left(1+\boldsymbol{v}^{ \pm}\right)^{-1}$ for plane stress; the argument, $\zeta$, of complex valued functions is not shown for compactness. The bi-holomorphic function $\mathbf{H}$ is the following combination of complex potentials

$$
\mathrm{H}(z, \bar{z})=\bar{z} \varphi^{\prime}(z)+\psi(z), \quad \mathrm{H}^{ \pm}=\lim _{z \rightarrow \zeta} \mathrm{H}(z, \bar{z}), \quad z \in \Omega^{ \pm}, \quad \zeta \in \Gamma
$$

Let the orientations of the displacements be known on the boundary while tractions are continuous across the contour. Then one arrives at the following boundary value problem for the holomorphic functions: Find two holomorphic functions $\varphi(z)$ and $\boldsymbol{\psi}(z)$ by the following boundary conditions on $\boldsymbol{\Gamma}$

$$
\begin{gathered}
T^{+}-T^{-}=0 \\
\arg W^{ \pm}=\beta^{ \pm}
\end{gathered}
$$

provided that two real valued functions $\boldsymbol{\beta}^{ \pm}=\boldsymbol{\beta}^{ \pm}(\zeta)$ are given on the contour and they satisfy the Hölder condition. 
It should be noted that (6) is equivalent to the condition of the continuity of the stress vector $\sigma=\sigma_{n}+i \sigma_{\mathrm{t}}$ across $\Gamma$ due to the following relationship

$$
\frac{\partial}{\partial \zeta} t^{ \pm}=\varphi^{\prime \pm}+\bar{\varphi}^{\prime \pm}+\frac{\partial \bar{\zeta}}{\partial \zeta}\left(\zeta \bar{\varphi}^{\prime \prime \pm}+\bar{\psi}^{\prime \pm}\right)=\sigma_{n}^{ \pm}+i \sigma_{t}^{ \pm}, \quad \varsigma \in \Gamma
$$

Boundary conditions (7) can also be expressed as follows:

$$
\operatorname{Im}\left(e^{-i \beta^{ \pm}} W^{ \pm}\right)=0
$$

As soon as the potentials are found, the stress fields (i.e., stress functions and stress components) in both exterior and interior domains can be determined by the Kolosov-Muskhelishvili formulas.

The sum of equations in (4) gives the following boundary values of the holomorphic function $\varphi$

$$
\left(\kappa^{ \pm}+1\right) \varphi^{ \pm}=2 G^{ \pm} W^{ \pm}+T^{ \pm}
$$

Let us denote for the jump of a quantity across the contour as $\langle f\rangle=f^{+}-f^{-}$. Then taking into account (6) one can present the jump of the holomorphic function $\varphi$ in the form

$$
\langle\varphi\rangle=\left\langle\frac{2 G}{\kappa+1} W\right\rangle+\left\langle\frac{1}{\kappa+1}\right\rangle T \equiv g
$$

where $T=T^{+}=T^{2}$.

As follows from (4)-(6) the jump of the holomorphic function $\psi$ is

$$
\langle\psi\rangle=-\langle\bar{\varphi}\rangle-\bar{\zeta}\left\langle\varphi^{\prime}\right\rangle \equiv h
$$

In view of the Cauchy integral formula one can present the holomorphic functions $\varphi$ and $\psi$ via the Cauchy integrals

$$
\varphi(z)=\frac{1}{2 \pi i} \int_{\Gamma} \frac{g(\eta) d \eta}{\eta-z}, \quad \psi(z)=\frac{1}{2 \pi i} \int_{\Gamma} \frac{h(\eta) d \eta}{\eta-z}, \quad z \in \Omega^{ \pm}
$$

Here, for simplicity, it is assumed that the potentials vanish at infinity (although the general case does not present significant difficulties).

The derivative of $\varphi$ is expressed via the Cauchy integrals with the density g'( $\eta)$. Then, as follows from (5), the bi-holomorphic function $\mathrm{H}$ is

$$
\mathrm{H}(z, \bar{z})=\frac{1}{2 \pi i} \int_{\Gamma} \frac{\bar{z} g^{\prime}(\eta)+h(\eta)}{\eta-z} d \eta, \quad z \in \Omega^{ \pm}
$$

The boundary values of $\varphi$ and its conjugate are found as follows:

$$
2 \varphi^{ \pm}= \pm g+\mathbf{S} g, \quad 2 \bar{\varphi}^{ \pm}= \pm \bar{g}+\overline{\mathbf{S} g}
$$

The function $h(\boldsymbol{\eta})$ is expressed via the function $g(\boldsymbol{\eta})$ as $h=-\bar{g}-\bar{\eta} g^{\prime}$ due to continuity of tractions, and the boundary values of $\mathbf{H}$ take the form

$$
2 \mathrm{H}^{ \pm}=\mp \bar{g}-\mathbf{S} \bar{g}+\mathbf{R}_{1} g
$$

where the regular operator $\mathbf{R}_{1}$ is

$$
\mathbf{R}_{1} g=-\frac{1}{\pi i} \int_{\Gamma} \frac{\bar{\eta}-\bar{\zeta}}{\eta-\zeta} g^{\prime}(\eta) d \eta=\frac{1}{\pi i} \int_{\Gamma}\left(\frac{d \bar{\eta} / d \eta}{\eta-\zeta}-\frac{\bar{\eta}-\bar{\zeta}}{(\eta-\zeta)^{2}}\right) g(\eta) d \eta
$$


In the latter formula one can notice that the argument is bounded and continuous on an arbitrary smooth contour. It also satisfies the Hölder condition, and its value at the singular point coincides with the angle of the tangent inclination to the real axis, thus

$$
\lim _{t \rightarrow \zeta} e^{-2 i \arg (t-\zeta)}=\lim _{t \rightarrow \zeta} \frac{\bar{t}-\bar{\zeta}}{t-\zeta}=\frac{d \bar{\zeta}}{d \zeta}=e^{-2 i \theta(\zeta)}
$$

In order to use directly the properties of SIE (1) it is necessary to recast the term $\mathbf{S} \bar{g}$ from (16) because it is not included into the dominant part of SIE (1). This can be done by applying the following property of the singular operator

$$
\overline{\mathbf{S} g}=-\mathbf{S} \bar{g}+\mathbf{R}_{2} \bar{g}
$$

where the regular operator $\mathbf{R}_{2}$ is

$$
\mathbf{R}_{2} \bar{g}=-\frac{1}{\pi i} \int_{\Gamma} \frac{\overline{g(\eta)}}{\eta-\zeta}\left(\frac{\eta-\zeta}{\bar{\eta}-\bar{\zeta}} \frac{d \bar{\eta}}{d \eta}-1\right) d \eta
$$

Its kernel is not singular, which is evident from (18).

Taking (19) into account one can rewrite (16) in the form

$$
2 \mathrm{H}^{ \pm}=\mp \bar{g}+\overline{\mathbf{S} g}+\mathbf{R}_{1} g-\mathbf{R}_{2} g, \quad 2 \overline{\mathrm{H}}^{ \pm}=\mp g+\mathbf{S} g+\overline{\mathbf{R}_{1} g}-\overline{\mathbf{R}_{2} g}
$$

\subsection{Complex SIE and its solvability}

Now let us assemble a system of real SIE by substituting (15) and (21) into the first eqn (4) followed by its substitution into (9) with results in

$$
\begin{gathered}
e^{-i \beta^{ \pm}}\left( \pm\left(\kappa^{ \pm}+1\right) g+\left(\kappa^{ \pm}-1\right) \mathbf{S} g\right)-e^{i \beta^{ \pm}}\left( \pm\left(\kappa^{ \pm}+1\right) \bar{g}+\left(\kappa^{ \pm}-1\right) \overline{\mathbf{S} g}\right)+\mathbf{r e g}^{ \pm}(g, \bar{g})=0 \\
\operatorname{reg}^{ \pm}(g, \bar{g})=e^{i \beta^{ \pm}}\left(\mathbf{R}_{1} g-\mathbf{R}_{2} g\right)-e^{-i \beta^{ \pm}}\left(\overline{\mathbf{R}_{1} g}-\overline{\mathbf{R}_{2} g}\right)
\end{gathered}
$$

Here the operators reg (.,.) are regular because $\mathbf{R}_{1}$ and $\mathbf{R}_{2}$ are regular. They satisfy the regular part in SIE (1) and it is evident that their real parts vanish.

The system of two real eqn (22) can be reduced to the form (1) by making their linear combination. In order to do that let us multiply these equations by two different complex functions $\exp \left(i \omega^{ \pm}\right)$respectively and then sum them up. This operation results in SIE (1) with the following coefficients.

$$
\begin{aligned}
& a=\left(\kappa^{+}+1\right) e^{-i\left(\beta^{+}-\omega^{+}\right)}-\left(\kappa^{-}+1\right) e^{-i\left(\beta^{-}-\omega^{-}\right)} \\
& b=-\left(\kappa^{+}+1\right) e^{i\left(\beta^{+}+\omega^{+}\right)}+\left(\kappa^{-}+1\right) e^{i\left(\beta^{-}+\omega^{-}\right)} \\
& c=\left(\kappa^{+}-1\right) e^{-i\left(\beta^{+}-\omega^{+}\right)}+\left(\kappa^{-}-1\right) e^{-i\left(\beta^{-}-\omega^{-}\right)} \\
& d=-\left(\kappa^{+}-1\right) e^{i\left(\beta^{+}+\omega^{+}\right)}-\left(\kappa^{-}-1\right) e^{i\left(\beta^{-}+\omega^{-}\right)}
\end{aligned}
$$

Substitution of (23) into (3) gives the following expression for the function $G$

$$
G=-e^{2 i \delta} \frac{\kappa^{+} \kappa^{-}-e^{-2 i \delta}}{\kappa^{+} \kappa^{-}-e^{2 i \delta}}, \quad \delta=\beta^{+}-\beta^{-}
$$


Note that this function does not depend on the angles $\omega^{ \pm}$, which means that those angles can be selected arbitrary except equal to $\pi \mathrm{k}$.

Since $\boldsymbol{\kappa}>1$ the fraction in (24) does not affect the index as its real part is always positive, therefore, the index is as follows:

$$
2 \mathrm{~K} \equiv \operatorname{Index}(G)=\frac{1}{2 \pi i}\left[\left.\ln (\exp (2 i \delta)]\right|_{\Gamma}=\left.\frac{\delta}{\pi}\right|_{\Gamma} .\right.
$$

The latter formula indicates that the index depends on the difference of the displacement orientations only, i.e. on the angle $\boldsymbol{\delta}$, whose increment (after the complete traverse of $\boldsymbol{\Gamma}$ in the positive direction) should be positive to provide a solution of the considered BVP. If $2 \mathrm{~K} \leq 0$, no bounded (non-trivial) solutions exist. For $2 \mathrm{~K}>0$ the solution of (22) includes an arbitrary polynomial of $2 \mathrm{~K}-1$ degree, which means that the solution is controlled by up to $4 \mathbf{K}$ real parameters. It should be noted that the degree of the polynomial (and the number of constants respectively) could be reduced if certain restrictions are introduced. This is shown in detail in the next section.

\section{NUMERICAL APPROACH}

Following Gakhov [1] we introduce the following operator

$$
\Theta(.)=\bar{a} \mathbf{I}(.)-b \overline{\mathbf{I}(.)}-\bar{c} \mathbf{S}(.)-d \overline{\mathbf{S}(.)}
$$

where $\mathbf{I}$ is the identical operator, i.e. $\mathbf{I} f=f$.

Let us apply this operator to the SIE of general type in (1). This allows one to exclude the complex conjugated variable in the dominant part of the resulting SIE that eventually assumes the form

$$
\begin{gathered}
A g+i B \mathbf{S} g+\mathbf{U} g+\overline{\mathbf{V} g}=0 \\
A=a \bar{a}-b \bar{b}-c \bar{c}+d \bar{d}, \quad i B=\bar{a} c-a \bar{c}+\bar{b} d-b \bar{d}, \quad \operatorname{Im}(A)=\operatorname{Im}(B)=0
\end{gathered}
$$

Here $\mathbf{U}$ and $\mathbf{V}$ are certain regular operators originated from the application of the operator $\boldsymbol{\Theta}$ and permutations of the singular and regular operators.

Let us seek the general solution of homogeneous SIE (27) as the sum of two unknown functions

$$
g=g_{0}+g_{1}
$$

Here $\mathrm{g}_{0}$ is the general solution of the homogeneous dominant equation

$$
A g_{0}+i B \mathbf{S} g_{0}=0
$$

and $g_{1}$ is any particular solution of the non-homogeneous SIE

$$
A g_{1}+i B \mathbf{S} g_{1}+\mathbf{U} g_{1}+\overline{\mathbf{V} g_{1}}=f_{0}
$$

where the right hand side is expressed through the solution of (29) in the form

$$
f_{0}=-\mathbf{U} g_{0}-\overline{\mathbf{V} g}
$$

The advantage of the presentation in (28) is evident from the fact that the direct attempt to solve (27) by any numerical method would produce a trivial solution only because of the homogeneous right hand side. On the other hand a particular numerical solution can be found for non-homogeneous SIE (30) as soon as $g_{0}$ becomes known. Therefore, the first step in the 
proposed numerical approach is to determine the full set of linearly independent solutions of SIE (29). Consider this operation in detail.

Firstly, let us reduce SIE (29) to the following Riemann problem by using the first formula in (15)

$$
(A+i B) \varphi^{+}-(A-i B) \varphi^{-}=0
$$

Further, by dividing (32) by $|A+i B|$ one finds

$$
\exp (+i \lambda) \varphi^{+}-\exp (-i \lambda) \varphi^{-}=0
$$

where the angle $\lambda$ is the argument of $A+i B$

$$
\lambda=\arg (A+i B)
$$

Let $\mathrm{K}$ be the index of the function $A-i B$ determined as the increment of the angle $-\lambda$ gained after the complete traverse of the contour $\Gamma$ counterclockwise, i.e. as follows:

$$
\mathrm{K}=\operatorname{Index}(A-i B)=\operatorname{Index}(\exp (-i \lambda))=-\left.\frac{1}{2 \pi}[\lambda]\right|_{\Gamma}
$$

Then one can reduce (33) to the following form

$$
\zeta^{\mathrm{K}} \exp (-i \lambda)\left(\zeta^{\mathrm{K}} \varphi^{+}\right)-\zeta^{\mathrm{K}} \exp (i \lambda)\left(\zeta^{\mathrm{K}} \varphi^{-}\right)=0
$$

Bearing in mind that $\operatorname{Index}\left(\zeta^{\mathbf{K}}\right)=\mathbf{K}$ we conclude that

$$
\operatorname{Index}\left[\zeta^{ \pm \mathrm{K}} \exp (\mp i \lambda)\right]=\left.\frac{1}{2 \pi i}[ \pm \mathrm{K} \ln \zeta \mp i \lambda]\right|_{\Gamma}=0
$$

This allows one to define a holomorphic function $\mu$ by the jump of its boundary values across the contour, i.e. via $\boldsymbol{\mu}^{+}-\boldsymbol{\mu}^{-}=\mathbf{K} \ln \zeta-i \lambda$. By the Plemelj-Sokhotskij formulas one can find the boundary values of this function in the form

$$
2 \mu^{ \pm}= \pm(\mathrm{K} \ln \zeta-i \lambda)+\mathbf{S}(\mathrm{K} \ln \zeta-i \lambda)
$$

Therefore

$$
\exp \left(2 \mu^{ \pm}\right)=\zeta^{ \pm \mathrm{K}} e^{ \pm i \lambda} \exp [\mathbf{S}(\mathrm{K} \ln \zeta-i \lambda)]
$$

Then (36) becomes

$$
\exp \left(2 \mu^{+}\right)\left(\zeta^{-\mathrm{K}} \varphi^{+}\right)=\exp \left(2 \mu^{-}\right)\left(\zeta^{\mathrm{K}} \varphi^{-}\right)
$$

Here, the exponential terms have a zero index while the term in parenthesis in the left hand side has a pole of order K-1 (as the value $\varphi^{+}(0)$ does not affect stresses we can assume $\left.\varphi^{+}(0)=0\right)$ and the term in parenthesis in the left hand side increase as $\zeta^{\mathrm{K}-1}$ at infinity (as we assumed that $\varphi^{-}(\infty) \rightarrow 0$ ). In accordance with the generalised Liouville's theorem [1] one can consider a holomorphic function $R$ in the entire plane that has the poles of order K-1 at the origin and at infinity, which solves the homogeneous Riemann problem. On the contour it has the form

$$
\exp \left(2 \mu^{+}\right)\left(\zeta^{\mathrm{K}} \varphi^{+}\right)=\exp \left(2 \mu^{-}\right)\left(\zeta^{\mathrm{K}} \varphi^{-}\right)=R
$$

where the expression for the holomorphic function $R$ is as follows:

$$
R(\zeta)=\frac{c_{-K+1}}{\zeta^{\mathrm{K}-1}}+\ldots \frac{c_{-1}}{\zeta}+c_{0}+c_{1} \zeta+\ldots c_{\mathrm{K}-1} \zeta^{\mathrm{K}-1}
$$


Therefore

$$
\varphi^{+}=\exp \left(-2 \mu^{+}\right) \zeta^{\mathrm{K}} R(\zeta), \quad \varphi^{-}=\exp \left(-2 \mu^{-}\right) \zeta^{-\mathrm{K}} R(\zeta)
$$

and consequently

$$
g_{0}=\left[\zeta^{\mathrm{K}} \exp \left(-2 \mu^{+}\right)-\zeta^{\mathrm{K}} \exp \left(-2 \mu^{-}\right)\right] R(\zeta)
$$

By substituting the boundary values of the functions $\exp \left(2 \mu^{ \pm}\right)$from (39) into the right hand side of (44) one can derive the final expression for the homogeneous solution in the form

$$
g_{0}=-2 i \sin (\lambda) \exp [-\mathbf{S}(\mathrm{K} \ln \zeta-i \lambda)] R(\zeta)
$$

The second step consists of finding particular numerical solutions of non-homogeneous SIE (30) where the right hand side is a linear combination of homogeneous solutions with arbitrary complex coefficients. In other words SIE (30) should be solved for $2 \mathrm{~K}-1$ different right hang sides, which can be written in the form

$$
A g_{1}^{j}-i B \mathbf{S}_{1}^{j}+\mathbf{U}_{1}^{j}+\overline{\mathbf{V}}_{1}^{j}=F_{j}, \quad j=0 \ldots 2 \mathrm{~K}-2
$$

Here the functions $F_{j}$ are expressed through the homogeneous solution, by taking into account (42) and (31), as follows:

$$
\begin{aligned}
& F_{j}(\zeta)=2 i \mathbf{U}\left[\sin (\lambda) \exp [-\mathbf{S}(\mathrm{K} \ln \zeta-i \lambda)] \zeta^{j-\mathrm{K}+1}\right]- \\
& -2 i \overline{\mathbf{V}\left[\sin (\lambda) \exp [-\mathbf{S}(\mathrm{K} \ln \zeta-i \lambda)] \zeta^{j-\mathrm{K}+1}\right]}, \quad j=0 \ldots 2 \mathrm{~K}-2
\end{aligned}
$$

It is evident that for calculation of the right hand side, it is necessary to perform two numerical integrations of two recurrent integrals. That is also an advantage over the method proposed earlier [4] where more computational efforts were needed. It should be noted that if $\mathbf{U}$ and $\mathbf{V}$ are complex conjugated operators (as in the case considered here, see (22)) than $F_{j}$ are real valued functions.

The general solution for $g_{1}$ is a linear combination of the particular solutions $g^{j}{ }_{1}$ with the coefficients $c_{j}$ in (42). It has the form

$$
g_{1}(\zeta)=\sum_{j=0}^{2 \mathrm{~K}-2} c_{j-K+1} g_{1}^{j}(\zeta)
$$

Combining the solutions for $g_{0}$ and $g_{1}$ one obtains the following final form for the general solution of SIE (27)

$$
g(\zeta)=\sum_{j=0}^{2 \mathrm{~K}-2} c_{j-K+1}\left[g_{1}^{j}(\zeta)-2 i \zeta^{j-K+1} \sin (\lambda) \exp [-\mathbf{S}(\mathrm{K} \ln \zeta-i \lambda)]\right]
$$

This solution is valid for any coefficients $a, b, c$ and $d$ that generate the functions $A$ and $B$. Let us present the expressions for the latter functions for the problem considered here by choosing $\omega^{ \pm}=-\beta^{ \pm}$, which yields

$$
\begin{aligned}
& a=\left(\kappa^{+}+1\right) e^{-2 i \beta^{+}}-\left(\kappa^{-}+1\right) e^{-2 i \beta^{-}}, \quad b=-\kappa^{+}+\kappa^{-} \\
& c=\left(\kappa^{+}-1\right) e^{-2 i \beta^{+}}+\left(\kappa^{-}-1\right) e^{-2 i \beta^{-}}, \quad d=-\kappa^{+}-\kappa^{-}+2
\end{aligned}
$$

Then the expressions for the coefficients of the Riemann problem assume the form

$$
A \mp i B=\mp 8 i \sin (\delta) e^{ \pm i \delta}\left(\kappa^{+} \kappa^{-}-e^{\mp 2 i \delta}\right), \quad \delta=\beta^{+}-\beta^{-}
$$


It is seen that Index $(A \pm i B)=\mp \mathrm{K}$ and therefore

$$
\operatorname{Index}\left(\frac{A-i B}{A+i B}\right)=2 \mathrm{~K}
$$

The latter formula agrees with the index calculated in (24)-(25).

\section{CONCLUSIVE REMARKS}

The BVP formulated in terms of the displacement vector orientations given on a contour separating dissimilar domains has been investigated for the case when the stress vector is continuous across the contour. It has been shown that for the positive index $2 \mathrm{~K}$ the solution includes a polynomial of up to $2 \mathrm{~K}-1$ degree with arbitrary complex coefficients. For the case of non-positive index no bounded solutions exist. This result is in line with that obtained for the similar materials in [2], which indicates that the elastic properties of the composed plane are not responsible for the number of linearly independent solutions.

One may expect obtaining closed form solutions for simple contours like circumferences or straight lines. This is due to the fact that the regular operators do not affect the problem, e.g. $\mathbf{R}_{1}=\mathbf{R}_{2}=0$ for half-planes. However, this simplification is not enough for finding the analytic form of the solution because the operators $\mathbf{U}$ and $\mathbf{V}$ do not vanish in general (although they can assume simple forms for specific functions $\beta$ ). It should be noted that if one assumes that the modulus of the displacement vectors are the same from different sides of the contour (for instance in the case of half-planes with the same elastic moduli) then the BVP can be decoupled, which indicates that in this case the boundary values of the displacement orientations cannot be chosen independently.

The numerical approach developed in this study has advantages over the previous approach suggested in [4] for the BVP formulated in terms of the boundary values of the principal directions given on both sides of the contour (assuming continuity of tractions). Such BVP was reduced to a SIE of the form (1). However the approach [4] utilized the Carleman-Vekua regularisation to transform the SIE into an integral equation of the Fredholm type, which requires more numerical integrations as compare to the approach reported here.

\section{ACKNOWLEDGEMENTS}

The author is thankful to the Russian Foundation for Basic Research that supported this work by the Grant 16-01-00260.

\section{REFERENCES}

[1] Gakhov, F.D., Boundary value problems. Moscow, Nauka, 1977, $3^{\text {rd }}$ Russian edition, see also the translation of the first edition by Pergamon Press, 1966.

[2] Galybin, A.N., Boundary value problems of plane elasticity involving orientations of displacements and tractions. Journal of Elasticity, 102, pp. 15-30, 2011. http://dx.doi.org/10.1007/s10659-010-9259-4

[3] Muskhelishvili, N.I., Some basic problems of the mathematical theory of elasticity. Springer-Verlag: Netherland, 1963.

[4] Galybin, A.N., Boundary integral equations for plane elastic problems posed in terms of stress orientations. Proceeding of the 8th UK Conference on Boundary Integral Methods, ed. D. Lesnic, University of Leeds, UK, July 2011. 